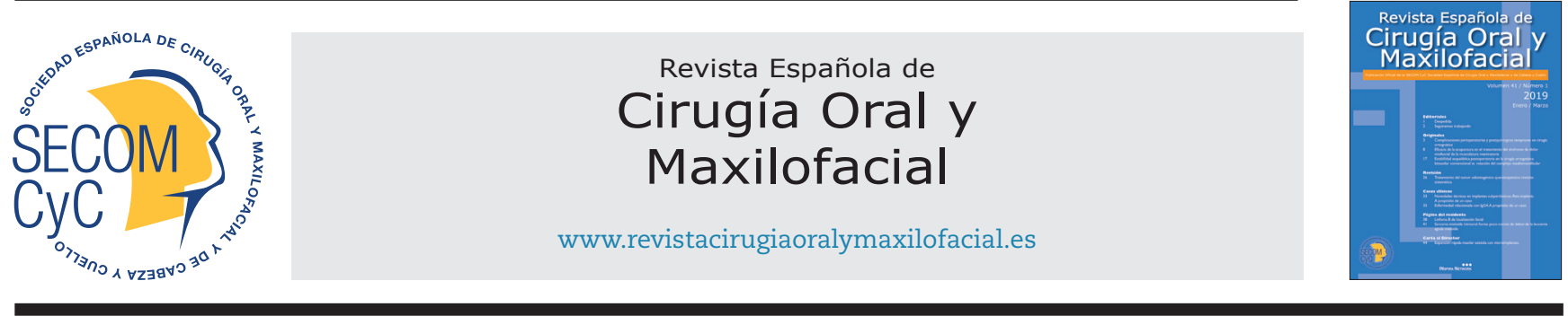

\title{
Original
}

\section{Complicaciones perioperatorias y postquirúrgicas tempranas en cirugía ortognática}

\author{
Jacinto Fernández Sanromán ${ }^{* a}$ y Sofía Muñiz Somoza ${ }^{b}$ \\ a Jefe de Servicio Cirugía Oral y Maxilofacial y ${ }^{b}$ DUE. Hospital Povisa, Vigo, España
}

INFORMACIÓN DEL ARTÍCULO

Historia del artículo:

Recibido el 09 de abril de 2018

Aceptado el 05 de septiembre de 2018

Palabras clave:

Cirugía ortognática, deformidad dentofacial, complicaciones en cirugía ortognática.

\section{R E S U M E N}

Objetivos: La cirugía ortognática ha experimentado un desarrollo significativo en las últimas décadas que la ha convertido en una técnica segura, pero no está exenta de presentar complicaciones. El objetivo del estudio es evaluar las complicaciones perioperatorias y postquirúrgicas inmediatas (durante el ingreso del paciente) asociadas a la cirugía ortognática en pacientes intervenidos de deformidades dentofaciales en nuestro hospital, analizando las características epidemiológicas de los pacientes y las posibles asociaciones entre el sexo, la edad, el tipo de osteotomía realizada y las complicaciones más frecuentes.

Material y métodos: Realizamos un estudio descriptivo retrospectivo que comprende 284 intervenciones de cirugía ortognática realizadas consecutivamente entre el 1 de enero del 2000 y el 31 de diciembre del 2016 en el Hospital Povisa de Vigo.

Resultados: La media de edad de los pacientes intervenidos fue de 28,72 años. El 64,4 \% eran mujeres. La deformidad dentofacial más frecuentemente tratada fue la maloclusión clase III de Angle, y la osteotomía con mayor incidencia fue la osteotomía tipo Le Fort I del maxilar superior. La mayoría de los pacientes fueron clasificados como ASA I. El tiempo operatorio promedio fue de 174 minutos y el tiempo medio de hospitalización fue de 3,26 días. Las náuseas o vómitos en el postoperatorio inmediato (NVPO) fue la complicación más frecuentemente registrada. Se encontraron correlaciones estadísticamente significativas entre el tipo de osteotomía realizada y la hemorragia, las NVPO, la ansiedad, la necesidad de transfusión sanguínea y el dolor postoperatorio.

Conclusión: El bajo índice de complicaciones encontradas en el presente estudio sugiere que la cirugía ortognática es un procedimiento quirúrgico seguro.

\footnotetext{
${ }^{*}$ Autor para correspondencia

Correo electrónico: jsanroman@povisa.es (Jacinto Fernández Sanromán).

https://doi.org/10.20986/recom.2019.1034/2019
}

1130-0558/@ 2019 SECOM. Publicado por Inspira Network. Este es un artículo Open Access bajo la licencia CC BY-NC-ND (http:// creativecommons.org/licenses/by-nc-nd/4.0/). 


\section{Keywords:}

Orthognathic surgery, dentofacial deformity, complications of orthognathic surgery.

\section{A B S T R A C T}

Objectives: Orthognathic surgery has undergone a significant development in recent years becoming a safe surgical technique. However, different complications can occur after orthognathic surgery. The purpose of this study is to analyze the possible complications found during surgery and immediately after orthognatic surgery, at our institution.

Material and methods: A retrospective descriptive study comprising 284 consecutive orthognathic surgical procedures performed between January 1, 2000 and December 31, 2016 at Povisa Hospital, Vigo, Spain, were studied.

Results: Mean age of the treated patients was 28.72 years. $64.4 \%$ were women. The most frequent dentofacial deformity diagnosed was Angle class III malocclusion and the most frequent performed osteotomy was Le Fort I maxillary osteotomy. The majority of patients were classified as ASA I. Mean operative time was 174 minutes and mean hospitalization time was 3.26 days. Postoperative nausea or vomiting (PONV) was the most frequent complication found. Statistically significant correlations were found between the type of osteotomy performed and postoperative bleeding, PONV, anxiety, need of blood transfusion and postoperative pain.

Conclusions: The low rate of complications found in the present study suggests that orthognathic surgery is a safe surgical procedure.

\section{Introducción}

La cirugía ortognática es la intervención quirúrgica encargada de corregir las deformidades maxilomandibulares, consiguiendo una oclusión final correcta y armonizando la cara del paciente ${ }^{1-3}$. Además, estas osteotomías pueden utilizarse para el abordaje y la resección de diferentes tumores craneomaxilofaciales y ser un arma terapéutica en el tratamiento de la apnea del sueño ${ }^{4-6}$.

Es un procedimiento electivo en el que los pacientes son intervenidos con el propósito de obtener un beneficio funcional y estético, pero también psicosocial ${ }^{4-7}$.

En la actualidad, la cirugía ortognática es un procedimiento seguro $5,6,8,9$. La seguridad que ofrece se debe a los importantes avances ocurridos en los métodos de diagnóstico, en la planificación del tratamiento y en las técnicas quirúrgicas y anestésicas. Estas mejoras han contribuido a aumentar la confianza de los pacientes, favoreciendo una mayor demanda de este tipo de procedimientos ${ }^{8}$.

Sin embargo, la cirugía ortognática no está exenta de algunas complicaciones, siendo las más frecuentemente reportadas: el dolor postoperatorio ${ }^{10-13}$, el edema postquirúrgico ${ }^{2,14,15}$, las hemorragias intra o postoperatorias $2,5,6,14,16,17$, las náuseas y vómitos postoperatorios ${ }^{18-21}$, la infección del sitio quirúrgi$\mathrm{CO}^{5,7,10,14,22,23}$, las alteraciones psicosociales ${ }^{2,9}$ y la recidiva de la deformidad dentofacial ${ }^{2,9,14}$. Sin embargo, el índice de complicaciones es bajo y los eventos graves que amenazan la vida del paciente tras la cirugía son poco frecuentes ${ }^{8,18}$.

El objetivo del presente estudio es analizar la incidencia de las complicaciones encontradas durante la cirugía y en el postoperatorio inmediato (durante el ingreso del paciente) en los pacientes intervenidos de cirugía ortognática en nuestro hospital y su posible relación con el tipo de osteotomía realizada, la edad y el sexo de los pacientes. No se estudian otras complicaciones que pueden aparecer en el postoperatorio tardío, como las alteraciones neurológicas, la recidiva de la deformidad, el retardo en la consolidación, etc.

\section{Material y métodos}

Se trata de un estudio observacional descriptivo retrospectivo, realizado en el Hospital Povisa de Vigo, España, que comprende la revisión de 284 intervenciones de cirugía ortognática realizadas consecutivamente en el Servicio de Cirugía Oral y Maxilofacial, entre el 1 de enero del 2000 y el 31 de diciembre del 2016

Se revisaron todas las historias clínicas informatizadas del hospital para la obtención de las diferentes variables analizadas: edad, sexo, tipo de deformidad dentofacial (clase II de Angle, clase III de Angle y otras: asimetría facial, mordida abierta anterior, etc.), tipo de osteotomía realizada (Le Fort I, osteotomía sagital de mandíbula, bimaxilar: maxilar y mandibular y genioplastia), clasificación ASA (American Society of Anesthesiogists: I-V), tiempo quirúrgico, tiempo de hospitalización, control de dolor postoperatorio, edema facial postoperatorio (clínico), presencia de náuseas o vómitos en el postoperatorio, ansiedad postoperatoria, necesidad de transfusión sanguínea (autodonación) en el postoperatorio, y otras (infección, reintervención). Todos los pacientes recibieron profilaxis antibiótica en la inducción anestésica, administración de $8 \mathrm{mg}$ de dexametasona y siguieron la misma guía clínica postoperatoria: cabecera elevada, aplicación de frío en la cara durante las primeras 12 horas, desketoprofeno $50 \mathrm{mg}$ cada 8 horas i.v., dexametasona $8 \mathrm{mg}$ cada 6 horas i.v.

Los datos han sido analizados con el programa estadístico IBM SPSS Statistics 15.0 para la obtención de las estadísticas descriptivas y la medición de las diferentes asociaciones entre las variables. 


\section{Resultados}

De los 284 pacientes intervenidos de cirugía ortognática durante el periodo de estudio, 183 fueron mujeres $(64,4 \%)$ y 101 hombres (35,6\%) de edades comprendidas entre 16 y 62 años. La media de edad de los pacientes fue de 28,72 \pm 9 años.

La deformidad dentofacial más común fue la maloclusión clase III de Angle, con un total de 134 pacientes afectados (47,2\%), seguida de la maloclusión clase II de Angle, con un total de 76 pacientes (26,8 \%), y de otras deformidades que, debido a su menor incidencia, se han categorizado bajo el término "otras" (asimetría facial, atrofia ósea, hipoplasia del maxilar superior, hiperplasia condílea, microsomía hemifacial, compresión transversal del maxilar, compresión mandibular, mordida abierta anterior (MAA), síndrome de cara larga y sonrisa gingival), con un total de 74 pacientes $(26,1 \%)$.

La osteotomía realizada con más frecuencia fue la del maxilar superior (27,8\%), efectuada en 79 casos, seguida de la bimaxilar (26,4\%), realizada en 75 casos, y de la mandibular (13\%), en 37 casos. Las osteotomías bimaxilar más genioplastia fue realizada en 30 casos (10,6 \%), la genioplastia sola en 19 casos $(6,7 \%)$ y la expansión rápida del paladar asistida quirúrgicamente o SARPE (6,3\%) en 18 casos. La osteotomía del maxilar superior más genioplastia $(5,6 \%)$ fue efectuada en 16 casos y, por último, la menos frecuente fue la osteotomía mandibular más genioplastia (3,5\%), realizada solo en 10 casos.

La mayoría de los pacientes intervenidos fueron clasificados como ASA I (53,9\%) y ASA II (42,3\%). Un pequeño porcentaje de pacientes fue catalogado como ASA III (3,5\%) y ASA IV $(0,4 \%)$.

El tiempo operatorio promedio fue de 174 minutos y el tiempo medio de hospitalización fue de 3,26 días.

En cuanto a las complicaciones encontradas, de los 284 pacientes estudiados, 223 (78,2 \%) presentaron dolor controlado con el tratamiento pautado en la guía clínica, mientras que los 61 restantes $(21,4 \%)$ manifestaron dolor no controlado, y requirieron rescate analgésico (metamizol i.v., acetaminofén i.v., meperidina i.v.). Los pacientes que experimentaron dolor no controlado fueron sometidos a: cirugía bimaxilar $(n=23)$, maxilar ( $n=15)$, bimaxilar con mentón $(n=12)$, mandibular $(n=7)$, mentoplastia $(n=2)$, cirugía mandibular con mentón $(n=1)$ y SARPE $(n=1)$. La osteotomía bimaxilar fue la que más se correlacionó con el dolor no controlado. Al relacionar el tipo de osteotomía con el dolor postoperatorio, se determinó una significación de $\mathrm{p}=0,007$ (Tabla I).

Respecto al edema postoperatorio, el 80,8 \% padeció inflamación leve-moderada y el resto sufrió inflamación severa. Los pacientes sometidos a cirugía bimaxilar y bimaxilar más genioplastia sufrieron edema severo con mayor frecuencia que el resto de los grupos este caso, severa ( $p=0,172$ ).

56 pacientes $(19,7 \%)$ presentaron hemorragia postoperatoria. Fueron transfundidos (autodonación) 35 pacientes (12,3\%). Los 249 pacientes restantes $(87,7 \%)$ no necesitaron ser transfundidos (Tabla II). Los pacientes que sufrieron hemorragia fueron sometidos a las siguientes osteotomías: bimaxilar $(n=24)$, maxilar ( $n=17)$, bimaxilar más genioplastia $(n=8)$, SARPE $(\mathrm{n}=4)$, cirugía mandibular $(\mathrm{n}=2)$ y maxilar más genioplastia $(\mathrm{n}=1)$. La osteotomía bimaxilar, con o sin genioplastia, fue la que más se correlacionó con la presencia de hemorragias
Tabla I. Resumen de las complicaciones encontradas en la muestra de pacientes intervenidos de cirugía ortognática

Complicaciones

Frecuencia Porcentaje

\begin{tabular}{lcc} 
Inflamación leve-moderada & 82 & $28,8 \%$ \\
Inflamación severa & 20 & $7 \%$ \\
Dificultades de la deglución & 13 & $4,6 \%$ \\
Mareos & 7 & $2,5 \%$ \\
Limitación importante de la & & \\
apertura oral & 3 & $1,1 \%$ \\
Equimosis del suelo de la boca & 3 & $1,1 \%$ \\
Dificultad respiratoria & 1 & $0,4 \%$ \\
Molestias en el ojo izquierdo & 1 & $0,4 \%$ \\
Sensación de taponamiento en el & & \\
oído derecho & 1 & $0,4 \%$ \\
Secreciones abundantes & 1 & $0,4 \%$ \\
Malestar general & 1 & $0,4 \%$ \\
Fotofobia & 1 & $0,4 \%$ \\
Parestesias & 1 & $0,4 \%$ \\
Falta de propiocepción & 1 & $0,4 \%$ \\
Equimosis geniana bilateral & 1 & $0,4 \%$ \\
Sensación de falta de aire & 1 & $0,4 \%$ \\
Inflamación de la conjuntiva del ojo & & \\
derecho & 1 & $0,4 \%$ \\
Hipotensión postoperatoria & 1 & $0,4 \%$ \\
\hline
\end{tabular}

Tabla II. Distribución de las unidades de sangre transfundidas

\begin{tabular}{ccc} 
Unidades de sangre transfundidas & Frecuencia & Porcentaje \\
\hline 0 & 249 & 87,7 \\
1 & 19 & 6,7 \\
2 & 11 & 3,9 \\
3 & 3 & 1,1 \\
4 & 1 & 0,4 \\
5 & 1 & 0,4 \\
Total & 284 & 100
\end{tabular}

asociadas a la cirugía ortognática. Tras analizar la relación entre el tipo de osteotomía y la hemorragia se determinó una significación de $\mathrm{p}=0,003$.

El porcentaje de náuseas y vómitos postoperatorios (NVPO) fue del 24,6 \%, viéndose afectados por esta complicación 70 pacientes. Estos pacientes que padecieron NVPO fueron intervenidos de: cirugía bimaxilar $(n=26)$, bimaxilar más genioplastia $(\mathrm{n}=13)$, maxilar $(\mathrm{n}=14)$, maxilar más genioplastia $(n=3)$, mandibular $(n=7)$, mandibular más genioplastia $(n=5)$ y SARPE $(n=2)$. La cirugía bimaxilar fue la que más se correlacionó con la NVPO. Tras analizar la relación entre el tipo de osteotomía y NVPO, se encontró una significación de p =0,001. 
En cuanto a la ansiedad postoperatoria, solo 22 pacientes intervenidos $(7,7 \%)$ la padecieron. Los pacientes que presentaron ansiedad fueron sometidos a las siguientes ostetotomías: bimaxilar $(n=13)$, bimaxilar más genioplastia $(n=4)$, mandibular $(\mathrm{n}=3)$, maxilar $(\mathrm{n}=1)$ y maxilar más genioplastia $(\mathrm{n}=$ 1). La osteotomía bimaxilar fue la que más se correlacionó con la presencia de ansiedad postoperatoria. Al analizar el tipo de osteotomía con la ansiedad postoperatoria se encontró una significación de $\mathrm{p}=0,006$.

De los 284 pacientes incluidos en la muestra de estudio, fueron reintervenidos quirúrgicamente 18 pacientes $(6,3 \%)$. En tres casos la reintervención se debió a hemorragia no controlada para revisar las osteotomías; en el resto de los casos por presentar alteraciones en la oclusión final postoperatoria.

Ningún paciente presentó infección del sitio quirúrgico tras la cirugía. Otras complicaciones encontradas fueron: dificultad en la deglución severa (12 casos), mareos (7 casos), lesiones labiales (6 casos) y queratitis/conjuntivitis ocular (3 casos).

No se encontraron correlaciones estadísticamente significativas entre el sexo, la edad y las complicaciones más frecuentes ( $p>0,05)$, pero sí entre el tipo de osteotomía realizada (bimaxilar) y la hemorragia ( $p=0,003)$, la NVPO $(p=0,001)$ la ansiedad ( $p=0,006)$, la necesidad de transfusión sanguínea $(p=0,005)$ y el dolor $(p=0,007)$.

\section{Discusión}

Al igual que en las series revisadas $5,15,18,20,24,25$, en este estudio las deformidades dentofaciales tratadas mediante cirugía ortognática son más frecuentes en pacientes del género femenino.

El promedio de edad de los pacientes intervenidos fue de 28,72 años. Los hallazgos encontrados en la literatura son similares $7,10,16,18,20,24,25$. Sin embargo, el resultado que más se equipara al reportado en el presente estudio es el presentado en el de Shinagawa y cols. ${ }^{15}$, en el que se informa de una media de edad de los pacientes estudiados de 29 años.

En el presente estudio, la deformidad dentofacial más común fue la maloclusión clase III de Angle (47,2 \%), que afectó a un total de 134 pacientes. Este resultado difiere de lo reportado en la bibliografía consultada. Así, Scariot y cols. ${ }^{24}$ encontraron que la anomalía más frecuente fue la deficiencia transversal maxilar. Sin embargo, Licéaga-Reyes y cols. ${ }^{25}$ publicaron que el prognatismo mandibular fue la deformidad facial más frecuentemente tratada, lo que coincide con los hallazgos del presente estudio.

Respecto al tipo de osteotomía realizada, en este estudio el $27,8 \%$ de los pacientes fueron sometidos a osteotomía del maxilar superior ( $\mathrm{n}=79$ ), el $26,4 \%$ a osteotomía bimaxilar ( $\mathrm{n}=75$ ) y el $13 \%$ a la mandibular ( $\mathrm{n}=37$ ), en tanto un porcentaje más pequeño de pacientes fue intervenido mediante técnicas segmentadas o combinadas.

$\mathrm{Al}$ igual que en este estudio, Scariot y cols. ${ }^{24} \mathrm{y}$ Shinagawa y cols. ${ }^{15}$ reportaron, en sus respectivos trabajos, que la osteotomía realizada con más frecuencia fue la del maxilar superior seguida de la bimaxilar y la mandibular. En la investigación de Silva y cols. ${ }^{18}$, la osteotomía ejecutada con más frecuencia fue la mandibular, mientras que en la de Phillips y cols. ${ }^{20}$ se reportó la osteotomía bimaxilar como la más frecuente.
En cuanto a la clasificación ASA, en el presente estudio, el $53,9 \%$ de los pacientes fueron clasificados como ASA I ( $\mathrm{n}=153)$, el $42,3 \%$ como ASA II $(n=120)$, el 3,5 \% como ASA III $(n=10) y$ el $0,4 \%$ como ASA IV $(n=1)$. Silva y cols. ${ }^{18}$ reportaron resultados similares: el 51,56 \% fueron catalogados como ASA I, el 45,33\% como ASA II y el 3,11 \% como ASA III. En cambio, en el estudio de Shinagawa y cols. ${ }^{15}$, solo se incluyeron pacientes clasificados como ASA I (62 \%) y ASA II (38\%). Existe, por tanto, un predominio de pacientes catalogados como ASA I y ASA II, probablemente porque este tipo de procedimientos se realiza, por lo general, en personas jóvenes sin patologías sistémicas significativas.

El tiempo operatorio promedio fue de 174 minutos. Si se analiza el resultado obtenido con los hallazgos presentados en otras series, se observa una duración de la intervención quirúrgica menor que en otras publicaciones ${ }^{10,15,20,24}$.

En cuanto al tiempo de ingreso hospitalario, el tiempo medio fue de 3,26 días, mientras que en estudios como el de Shinagawa y cols. ${ }^{15}$, se reporta un tiempo de ingreso medio de 2 días. El tiempo de ingreso sin embargo ha ido reduciéndose en los últimos años en nuestro hospital, realizándose algunas técnicas sin ingreso hospitalario en el momento actual.

Se determinaron diferentes complicaciones asociadas a la cirugía ortognática. Al igual que en el estudio de Shinagawa y cols. ${ }^{15}$, las náuseas y vómitos postoperatorios (NVPO) constituyeron la complicación más frecuente $(24,6 \%)$, viéndose afectados 70 pacientes. Silva y cols. ${ }^{18}$ reportaron que 206 de los 514 pacientes analizados en su estudio padecieron NVPO (40,08\%), mientras que Donoso-Hofer y cols. ${ }^{22}$ observaron un porcentaje de NVPO del 33,3 \% que afectó a 24 pacientes. Las NVPO siguen siendo frecuentes tras la cirugía ortognática a pesar de tomar medidas preventivas como realizar taponamiento posterior durante la cirugía, la aspiración gástrica al final del procedimiento quirúrgico y el uso de fármacos antieméticos. El uso de fármacos antieméticos y la suspensión de la tolerancia oral permite el control de las mismas, que no suelen ocurrir después de las primeras 24 horas de la cirugía.

En este estudio no se registró ningún caso de infección del sitio quirúrgico (ISQ) durante el periodo postoperatorio. En los estudios de Shinagawa y cols. ${ }^{15}$ y Donoso-Hofer y cols. ${ }^{10}$ se reportó infección del sitio quirúrgico como evento adverso postoperatorio, probablemente por el aumento del tiempo operatorio que ejerce una influencia negativa en el riesgo de ISQ incrementando la exposición a patógenos y el trauma de los tejidos. Quizás el uso de profilaxis antibiótica en cirugía ortognática pueda también a contribuir en la ausencia de infecciones postquirúrgicas en este tipo de pacientes.

Al igual que en otros estudios consultados ${ }^{5,15,24}$, la hemorragia fue uno de los eventos adversos más comunes asociados a la cirugía ortognática. En este estudio fueron transfundidos 35 pacientes (12,3\%), mientras que en el de Licéaga-Reyes y cols. ${ }^{25}$ fueron transfundidos tres. Esta disimilitud se debe, probablemente, a la diferencia en el tamaño muestral de ambos estudios. La hemorragia postoperatoria es significativamente más frecuente en aquellos pacientes intervenidos mediante múltiples osteotomías (bimaxilar o bimaxilar más genioplastia). La mejora en las técnicas anestésicas con hipotensión controlada ha permitido en los últimos años una reducción significativa en la necesidad de realizar transfusiones en el postoperatorio, por lo que en nuestro centro se ha abandonado hace años la realización de protocolos de autodonación, salvo en casos muy seleccionados. 
En cuanto a la reintervención quirúrgica, en este estudio fueron reintervenidos 18 pacientes (6,3\%). En el mismo estudio de Licéaga-Reyes y cols. ${ }^{25}$ se reportaron 10 pacientes $(9,3 \%)$ que requirieron reintervención como consecuencia de maloclusión o hemorragia postoperatorias.

En este estudio no se encontraron correlaciones estadísticamente significativas entre el sexo, la edad y las complicaciones más frecuentes, con lo cual estos parámetros no suponen un mayor riesgo de presentar complicaciones asociadas a la cirugía ortognática.

En el presente estudio, al relacionar el tipo de osteotomía y las complicaciones más frecuentes, sí se encontró una correlación estadísticamente significativa entre la osteotomía realizada (bimaxilar) y hemorragia ( $p=0,003)$, NVPO $(p=0,001)$, ansiedad $(p=0,006)$ y necesidad de transfusión sanguínea $(p=0,005)$ $y$ dolor $(p=0,007)$. Hay algunos autores ${ }^{16}$ que, sin embargo, no demuestran correlación estadísticamente significativa entre la necesidad transfusional y el tipo de cirugía más compleja.

\section{Conclusiones}

En este estudio se han reportado diferentes complicaciones asociadas a la cirugía ortognática, pero su incidencia es baja y la presencia de eventos graves que supongan un riesgo vital para el paciente inexistente. Por tanto, se puede considerar que la cirugía ortognática es un procedimiento quirúrgico seguro; además de predecible, como resultado de la planificación preoperatoria y los avances en las técnicas quirúrgicas y anestésicas. El conocimiento de estas complicaciones permite que el equipo asistencial mejore su prevención y detección precoz para que, en caso de que se presenten estos eventos, el manejo sea el adecuado.

\section{B I B L I O G R A F Í A}

1. Raspall G. Cirugía Maxilofacial. Patología quirúrgica de la cara, boca, cabeza y cuello. $1^{\mathrm{a}}$ ed. Madrid: Editorial Médica Panamericana; 2002.

2. Rodrigues-Machado M, Silva-Sousa C, Turrini RNT. La percepción de los pacientes sometidos a cirugía ortognática sobre el cuidado postoperatorio. Rev Esc Enferm USP. 2012;46:78-85. DOI: 10.1590/S0080-62342012000700012.

3. Vargas-Vargas R. Incidencia de complicaciones en pacientes con deformidad dentofacial sometidos a cirugía ortognática en el centro médico Adolfo López Mateos en un periodo de 5 años [Tesis doctoral]. México: Universidad Autónoma del Estado de México, Facultad de Medicina; 2013. Disponible en: http://ri.uaemex.mx/ bitstream/handle/20.500.11799/13821/413885.pdf?sequence=1.

4. León M, Lozano E, Moreno S. Cirugía ortognática: revisión de la literatura. Rev Estomatol. 2006;14(2):18-21.

5. Kim SG, Park SS. Incidence of Complications and Problems Related to Orthognathic Surgery. J Oral Maxillofac Surg. 2007;65(12):2438-44. DOI: 10.1016/j.joms.2007.05.030.

6. Morales-Trejo B. Complicaciones en cirugía ortognática. Conceptos actuales y revisión de la literatura. Rev ADM. 2015;72(5):230-5.

7. Davis CM, Gregoire CE, Steeves TW, Demsey A. Prevalence of Surgical Site Infections Following Orthognathic Surgery: A Retrospective Cohort Analysis. J Oral Maxillofac Surg. 2016;74(6):1199-206. DOI: 10.1016/j.joms.2016.01.040.

8. Hueto-Madrid JA, Gutiérrez-Santamaría J. Complicaciones quirúrgicas de la cirugía ortognática: presentación de tres casos y revisión de la literatura. Rev Esp Cir Oral Maxilofac. 2012;34(2):56-74. DOI: 10.1016/j.maxilo.2011.09.009.
9. Steel BJ, Cope MR. Unusual and Rare Complications of Orthognathic Surgery: A Literature Review. Oral Maxillofac Surg. 2012;70(7):1678-91. DOI: 10.1016/j.joms.2011.05.010.

10. Donoso-Hofer T, Villanueva-Maffei J, Araya-Cabello I, YanineMontaner N. Riesgo de infección del sitio quirúrgico, según tiempo operatorio en cirugía maxilofacial mayor limpia contaminada: estudio observacional analítico. Rev Clin Periodoncia Implantol Rehabil Oral. 2015;8(3):203-7. DOI: 10.1016/j. piro.2015.07.001.

11. Cosmelli-Maturana R, Cifuentes-Fernández J, Sepúlveda-Voullieme P. Dolor Postoperatorio en Cirugía Ortognática. Rev Dent Chile. 2008;99(2):17-22.

12. Aoki Y, Yoshida K, Nishizawa D, Kasai S, Ichinohe T, Ikeda $K$, et al. Factors that Affect Intravenous Patient-Controlled Analgesia for Postoperative Pain Following Orthognathic Surgery for Mandibular Prognathism. PLoS One. 2014;9(6):e98548. DOI: 10.1371/journal.pone.0098548.

13. Dávila-Cordero U, Cazar-Almache M, Viteri-León E. Analgesia multimodal postoperatoria en cirugía ortognática. Rev Mex Cir Bucal Max. 2015;11(2):45-7.

14. Duque FL, Jaramillo PM. Complicaciones asociadas con osteotomía Le Fort I. Rev Fac Odontol Univ Antioq. 2009;20(2):20521.

15. Shinagawa A, Melhem FE, De Campos AC, Dias-Cicarelli D, Frerichs E. Predictores de dolor y tiempo de internación prolongado tras cirugía ortognática: estudio de cohorte retrospectivo. Rev Colomb Anestesiol. 2015;43(2):129-35. DOI: 10.1016/j. rca.2015.01.004.

16. Stavaru-Marinescu B, Sastre-Pérez J, Fernández-Díaz JO, García-Jiménez T, Naval-Gías L, Díaz-González FJ. ¿Podemos predecir la pérdida de sangre en la cirugía ortognática utilizando parámetros preoperatorios? Rev Esp Cir Oral Maxilofac. 2013;35(3):116-22. DOI: 10.1016/j.maxilo.2012.06.001.

17. Hernández-Alfaro F, Rosario-Regalado R, Mair D. Autotransfusión sanguínea en cirugía ortognática: no necesario. Rev Esp Cir Oral Maxilofac. 2006;28(6):333-8.

18. Silva A, O’Ryan F, Poor D. Postoperative Nausea and Vomiting (PONV) After Orthognathic Surgery: A Retrospective Study and Literature Review. J Oral Maxillofac Surg. 2006;64(9):1385-97. DOI: 10.1016/j.joms.2006.05.024.

19. Moreira-Schmitt AR, Gambôa-Ritto F, Rodrigues Luz de Azevedo JG, D'Albuquerque-Medeiros PJ, Mattos de Mesquita MC. Efficacy of Gastric Aspiration in Reducing Postoperative Nausea and Vomiting After Orthognathic Surgery: A Double-Blind Prospective Study. J Oral Maxillofac Surg. 2017;75(4):701-8. DOI: 10.1016/j.joms.2016.10.002.

20. Phillips C, Brookes CD, Rich J, Arbon J, Turvey TA. Postoperative nausea and vomiting following orthognathic surgery. Int J Oral Maxillofac Surg. 2015;44(6):745-51. DOI: 10.1016/j. ijom.2015.01.006.

21. Acosta-Villegas F, García-López JA, Aguayo-Albasini JL. Manejo de las náuseas y vómitos postoperatorios. Cir Esp. 2010;88(6):369-73. DOI: 10.1016/j.ciresp.2010.07.009.

22. Salmerón-Escobar JI, del Amo- Fernández de Velasco A. Profilaxis antibiótica en Cirugía Oral y Maxilofacial. Med Oral Patol Oral Cir Bucal. 2006;11(3):292-6.

23. Christabel A, Sharma R, Manikandhan R, Anantanarayanan $P$, Elavazhagan N, Subash P. Fever After Maxillofacial Surgery: A Critical Review. J. Maxillofac. Oral Surg. 2015;14(2):154-61. DOI: 10.1007/s12663-013-0611-7.

24. Scariot R, da Costa DJ, Barbosa-Rebellato NL, Müller PR, da Conceição-Ferreira R. Epidemiological analysis of orthognathic surgery in a hospital in Curitiba, Brazil: review of 195 cases. Rev Esp Cir Oral Maxilofac. 2010;32(4):147-91. DOI: 10.1016/ S1130-0558(10)70034-4.

25. Licéaga-Reyes R, Trujillo-Fandiño JJ, Licéaga-Escalera CJ, Montoya-Pérez LA, Rodríguez-Ramírez E. Cirugía Ortognática en el Hospital Juárez de México. Análisis de 163 casos operados de 2007 a 2010. Rev Mex Cir Bucal Max. 2012;8(1):10-4. 